TRANSACTIONS OF THE

AMERICAN MATHEMATICAL SOCIETY

Volume 361, Number 6, June 2009, Pages 3071-3082

S 0002-9947(08)04743-0

Article electronically published on December 29, 2008

\title{
TOPOLOGICAL ENTROPIES OF EQUIVALENT SMOOTH FLOWS
}

\author{
WENXIANG SUN, TODD YOUNG, AND YUNHUA ZHOU
}

\begin{abstract}
We construct two equivalent smooth flows, one of which has positive topological entropy and the other has zero topological entropy. This provides a negative answer to a problem posed by Ohno.
\end{abstract}

\section{INTRODUCTION}

Two flows defined on a smooth manifold are equivalent if there exists a homeomorphism of the manifold that sends each orbit of one flow onto an orbit of the other flow while preserving the time orientation. The topological entropy of a flow is defined as the entropy of its time- 1 map. While topological entropy is an invariant for equivalent homeomorphisms (Theorem 7.2 in [13), finite non-zero topological entropy for a flow cannot be an invariant because its value is affected by time reparameterization. However, 0 and $\infty$ topological entropy are invariants for equivalent flows without fixed points (see [7, 8], [10, 11]).

In equivalent flows with fixed points there exists a counterexample, constructed by Ohno [7, showing that neither 0 nor $\infty$ topological entropy is preserved by equivalence. The two flows constructed in [7] are suspensions of a transitive subshift and thus are not differentiable. Note that a differentiable flow on a compact manifold cannot have $\infty$ entropy (see Theorem 7.15 in [13). These facts led Ohno [7] in 1980 to ask the following:

\section{Is 0 topological entropy an invariant for equivalent differentiable flows?}

In this paper, we construct two equivalent $C^{\infty}$ smooth flows with a singularity, one of which has positive topological entropy while the other has zero topological entropy. This gives a negative answer to Ohno's question.

We begin by supposing that $f: M \rightarrow M$ is a $C^{\infty}$ diffeomorphism of a smooth compact Riemannian manifold $M$ with $\operatorname{dim} M=m \geq 2$ with the following properties: (1) $f$ has positive topological entropy and (2) $f$ is minimal in the sense that all forward orbits are dense in $M$ (or equivalently closed invariant sets are either empty or the entire space). An example of such an $f$ was constructed by Herman [3. Using the constant function $I: M \rightarrow \mathbb{R}, I(x) \equiv 1$, one gets a suspension manifold $\Omega$

Received by the editors May 31, 2007.

2000 Mathematics Subject Classification. Primary 37C15, 34C28, 37A10.

Key words and phrases. Measure-theoretic entropy, equivalent flow, singularity.

The first author was supported by NSFC (\#10231020, \#10671006) and National Basic Research Program of China (973 Program) (\#2006CB805900).

The second author was supported by an Ohio University Faculty Fellowship Leave.

The third author was supported by NSFC (\#10671006). 
and a smooth suspension flow $\psi: \Omega \times \mathbb{R} \rightarrow \Omega$ (Definition 2.1). Let $X$ denote the smooth vector field associated with this flow. For any function $\alpha \in C^{\infty}(\Omega,[0,1])$, note that $\alpha X$ is a $C^{\infty}$ vector field on $\Omega$ and it thus induces a unique, differentiable flow.

Theorem 1.1 (Main Theorem). There exist two functions $\alpha, \widehat{\alpha} \in C^{\infty}(\Omega,[0,1])$ satisfying the following:

(1) $\alpha X$ and $\widehat{\alpha} X$ induce equivalent flows $\varphi(t)$ and $\widehat{\varphi}(t)$;

(2) $\varphi(t)$ has zero topological entropy and $\widehat{\varphi}(t)$ has positive topological entropy.

The key idea used by Ohno and in this work is that the time reparameterization between an orbit in one flow and its image orbit in an equivalent flow can grow super-exponentially near a fixed point. In the present case the main challenge is to ensure that the time parameterizations lead to differentiable flows. Throughout the rest of the paper we fix one point $p=\left(x_{0}, 0\right) \in \Omega$ and consider functions $\alpha, \widehat{\alpha} \in C^{\infty}(\Omega,[0,1])$ satisfying the following criteria:

$(\mathbf{H})$. We assume that $\alpha(p)=\widehat{\alpha}(p)=0, \alpha(q)$ and $\widehat{\alpha}(q)>0$, for $q \neq p \in \Omega$, and there exists a small neighborhood $V$ of $p$ in $\Omega$ such that $\alpha(q)=\widehat{\alpha}(q) \equiv 1$ if $q \in \Omega \backslash V$.

In our construction $\hat{\varphi}$ will be shown to have an ergodic probability measure that is equivalent to an ergodic probability preserved by the suspension flow of $f$. Positive entropy of $f$ will then imply positive entropy for $\widehat{\varphi}$. On the other hand, in the construction of $\varphi, \alpha$ will be very flat so that orbits near $p$ will be strongly slowed down. This together with minimality, which ensures fast returns to any neighborhood of $p$, will imply that $\phi$ has Dirac measure at $p$ as its unique ergodic probability measure.

\section{Preliminaries}

2.1. Notation. The symbol $\langle\cdot, \cdot\rangle$ will be used to denote the Riemannian metric on either $M$ or $\Omega$ depending on the context. We will denote a ball in $M$ by $B_{M}(x, \epsilon)=\{y \in M: d(x, y)<\epsilon\}$ and by $B_{\Omega}(x, \epsilon)$ a ball in $\Omega$. A ball in $\mathbb{R}^{n}$ centered at the origin we will denote by $B^{n}(r)=\left\{x \in \mathbb{R}^{n}:|x|<r\right\}$.

Definition 2.1. Suppose that $M$ is a smooth compact Riemannian manifold and that $f$ is a $C^{\infty}$ diffeomorphism. Consider the space

$$
\Omega=M \times[0,1] / \sim,
$$

where $\sim$ is the identification of $(y, 1)$ with $(f(y), 0)$. The standard suspension of $f$ is the flow $\psi_{t}$ on $\Omega$ defined by $\psi_{t}(y, s)=(y, t+s)$, for $0 \leq t+s<1$.

A standard argument as in [5] shows that $\Omega$ is a smooth compact Riemannian manifold and $\psi_{t}$ is $C^{\infty}$. If $f: M \rightarrow M$ is minimal as a homeomorphism, then $\psi_{t}$ is a minimal flow.

Proposition 2.2. If $\alpha$ and $\widehat{\alpha}$ in $C^{\infty}(\Omega,[0,1])$ satisfy $(\mathbf{H})$ and $X$ is the suspension vector field on $\Omega$ described above, then $\alpha X$ and $\widehat{\alpha} X$ induce equivalent differential flows on $\Omega$ with one singularity $p$.

Proof. That a $C^{\infty}$ vector field induces a $C^{\infty}$ flow is a standard result. The identity map on $\Omega$ takes orbits of one flow to orbits of the other since the unique singular point $p$ is mapped to itself and elsewhere $\alpha$ and $\widehat{\alpha}$ are positive. The assumption that $\alpha$ and $\widehat{\alpha}$ are non-negative also implies preservation of time orientation. 
2.2. Minimal homeomorphisms and uniform recurrence. The following result concerning uniform recurrence of orbits in minimal homeomorphisms will be used in the proof that $\varphi$ has zero entropy. It guarantees fast return of orbits to a neighborhood of the stopped point $p$.

Lemma 2.3. Let $M$ denote a smooth compact Riemannian manifold and let $f$ : $M \rightarrow M$ be a $C^{\infty}$ diffeomorphism. Suppose $(M, f)$ is a minimal homeomorphism. Then for any $\varepsilon>0$, there exists $L(\varepsilon) \in \mathbb{N}$ such that, for any $x, y \in M, f^{l}(y) \in$ $B(x, \varepsilon)$, for some $l, 0 \leq l \leq L(\varepsilon)$.

Proof. This is a classical result (see 2]). We present a proof for the convenience of the readers.

For any $x, y \in M$ and $\epsilon>0$, there exists $l(x, y, \varepsilon) \in \mathbb{N}$ such that $f^{l(x, y, \varepsilon)}(y) \in$ $B\left(x, \frac{\varepsilon}{2}\right)$, since $f$ is minimal. It follows that there exists $\delta(y)>0$ such that $f^{l(x, y, \varepsilon)}(B(y, \delta(y))) \subset B\left(x, \frac{3 \varepsilon}{4}\right) \subset B(x, \varepsilon)$. By the compactness of $M, \exists y_{1}, \ldots, y_{n}$ and $L(x, \varepsilon)=\max _{i}\left\{l\left(x, y_{i}, \varepsilon\right)\right\}$ such that

(i) $\bigcup_{i=1}^{n} B\left(y_{i}, \delta\left(y_{i}\right)\right)=M$;

(ii) $\forall y \in M, \exists l(y) \leq L(x, \varepsilon)$ such that $f^{l(y)}(y) \in B\left(x, \frac{3 \varepsilon}{4}\right) \subset B(x, \varepsilon)$.

Then, for any $\widetilde{x} \in B\left(x, \frac{\varepsilon}{4}\right), f^{l(y)}(y) \in B(\widetilde{x}, \varepsilon)$. The compactness of $M$ implies that there exist finite points $x_{1}, \ldots, x_{n_{0}}$ such that $\bigcup_{i=1}^{n_{0}} B\left(x_{i}, \frac{\varepsilon}{4}\right)=M$. Then, $L(\varepsilon)=\max _{i}\left\{L\left(x_{i}, \varepsilon\right)\right\}$ satisfies the lemma.

Uniform recurrence as in Lemma 2.3 allows us to put lower bounds on the measures of sets with respect to ergodic measures.

Lemma 2.4. Suppose the assumptions of Lemma 2.3 and suppose that $\mu$ is an ergodic measure of $f$. Then

$$
\mu\left(B_{M}(x, \varepsilon)\right) \geq \frac{1}{L(\varepsilon)}>0, \quad \forall x \in M, \varepsilon>0,
$$

where $L(\varepsilon)$ is as in Lemma 2.3 .

Proof. Set

$$
Q_{\mu}(f)=\left\{x \in M: \lim _{n \rightarrow \pm \infty} \frac{1}{n} \sum_{k=0}^{n-1} \xi\left(f^{k} x\right)=\int_{M} \xi(x) d \mu(x), \quad \forall \xi \in C^{0}(M)\right\} .
$$

By the Birkhoff ergodic theorem, $Q_{\mu}(f)$ is an $f$-invariant and $\mu$-full measure set. For any $x \in M$ and $\varepsilon>0$, take $L(\varepsilon)$ as in Lemma 2.3. If $y \in Q_{\mu}(f)$, then by the Birkhoff ergodic theorem and Lemma 2.3.

$$
\begin{aligned}
\mu\left(B_{M}(x, \varepsilon)\right) & =\lim _{n \rightarrow \infty} \frac{\operatorname{Card}\left\{i \in\{0,1, \ldots, n-1\}: f^{i}(y) \cap B_{M}(x, \varepsilon) \neq \emptyset\right\}}{n} \\
& \geq \frac{1}{L(\varepsilon)}>0 .
\end{aligned}
$$

2.3. Time-changed systems and entropy. First, we recall the notion of an additive function.

Definition 2.5. Suppose $\psi_{t}$ is a measurable flow on a Borel probability space $(\Omega, \mathcal{B}, \mu)$ and $\Omega$ is divided into disjoint invariant measurable sets $A$ and $N$ such that $\mu(A)=1$ and $\mu(N)=0$. Further suppose that $\theta(t, x)$ is a real measurable 
function defined on $(-\infty,+\infty) \times(\Omega \backslash N)=\mathbb{R} \times A$ with the following properties for every fixed $x \in A$ :

(1) $\theta(t, x)$ is continuous and non-decreasing in $t$;

(2) $\theta(t+s, x)=\theta(s, x)+\theta\left(t, \psi_{s} x\right)$ for all $t$ and $s$;

(3) $\theta(0, x)=0, \lim _{t \rightarrow \infty} \theta(t, x)=\infty, \lim _{t \rightarrow-\infty} \theta(t, x)=-\infty$.

Then $\theta$ is called an additive function of $\psi_{t}$ with carrier $A$. An additive function is said to be integrable if it is integrable in $\Omega$ for every fixed $t$.

Lemma 2.6. If $\psi_{t}$ is a measurable flow on a Borel probability space $(\Omega, \mathcal{B}, \mu)$ and $a(x)$ is a non-negative, integrable function satisfying

$$
E_{\mu}(a)=\int_{\Omega} a(x) d \mu(x)>0
$$

then the function

$$
\theta(t, x)=\int_{0}^{t} a\left(\psi_{s} x\right) d s
$$

is an integrable additive function.

For a proof see Theorem 3.1 in [12].

Definition 2.7. The function $\theta(t, x)$ in Lemma 2.6 is called the additive function defined by $a(x)$.

Let $\psi_{t}$ be a measurable flow on a Borel probability space $(\Omega, \mathcal{B}, \mu)$ and let $\theta$ be an additive function of $\psi_{t}$. Define

$$
\varphi_{t} x=\psi_{\tau(t, x)} x, \quad \text { with } \quad \tau(t, x)=\sup \{s: \theta(s, x) \leq t\},
$$

for all $-\infty<t<\infty$ and all $x \in A$, where $A$ is the carrier of $\theta$. We call $\widehat{\Omega}=\{x \in$ $A: \theta(t, x)>0, \forall t>0\}$ the regular set of $\theta$, in other words, the set of non-singular points of $\varphi_{t}$. Let $\widehat{\mathcal{B}}=\mathcal{B} \cap \widehat{\Omega}$. Then $\varphi_{t}:(\widehat{\Omega}, \widehat{\mathcal{B}}) \rightarrow(\widehat{\Omega}, \widehat{\mathcal{B}})$ is a measurable flow (see Lemma 4.1 in [12]), which is called the time changed system of $\psi_{t}$ by $\theta$.

Let $\mu$ be a $\psi_{t}$ invariant probability measure and $a(x)$ be a non-negative, integrable function. Define

$$
\widehat{\mu}(B)=\int_{B} d \widehat{\mu}(x)=\int_{B} a(x) d \mu(x)
$$

for all $B \in \mathcal{B}$. We get an invariant measure $\widehat{\mu}$ of the time changed system $\varphi_{t}$ (see ([6]).

Lemma 2.8 ([12]). If $\psi_{t}$ is ergodic, then any time changed flow $\varphi_{t}$ of $\psi_{t}$ is also ergodic.

The following is Theorem 10.1 of [12].

Theorem 2.9. Let $\psi_{t}$ be an arbitrary measurable flow on a Borel probability space $(\Omega, \mathcal{B}, \mu)$, and let $\theta$ be any integrable additive function of $\psi_{t}$. If the flow $\varphi_{t}$ on $(\widehat{\Omega}, \widehat{\mathcal{B}}, \widehat{\mu})$ is the time changed flow of $\phi_{t}$ by $\theta$, then we have the inequality

$$
h_{\widehat{\mu}}\left(\varphi_{t}\right) \widehat{\mu}(\widehat{\Omega}) \leq h_{\mu}\left(\psi_{t}\right) \mu(\Omega)
$$

for all fixed $t$, where $h_{\widehat{\mu}}\left(\varphi_{t}\right)$ and $h_{\mu}\left(\psi_{t}\right)$ denote the measure-theoretic entropies of the homeomorphisms $\varphi_{t}$ and $\psi_{t}$, respectively. The equality holds when $\psi_{t}$ is ergodic. 
This theorem will be used to establish that the entropy of $\widehat{\varphi}$ is non-zero, by exhibiting an ergodic $\mu$ with positive entropy and establishing that $\hat{\mu}$ is finite.

Definition 2.10. An ergodic measure is atomic if it is supported on a periodic orbit.

Lemma 2.11. Assume that $\psi_{t}$ is the standard suspension of $f$ from above, $X$ is the vector field that induces $\psi_{t}$ and $\alpha \in C^{1}(\Omega,[0,1])$ satisfies $(\mathbf{H})$ (see $\left.\S 1\right)$. Denote by $\varphi_{t}$ the flow induced by the vector field $\alpha X$ on $\Omega$. For any $x \in M$, define $\gamma(x)$ by:

$$
\begin{cases}\varphi_{\gamma(x)}(x, 0)=\psi_{1}(x, 0)=(f(x), 0), & (x, 0) \neq f^{-1}(p) \text { and }(x, 0) \neq p \\ \gamma(x)=+\infty, & (x, 0)=f^{-1}(p) \text { or }(x, 0)=p\end{cases}
$$

Then for any non-atomic ergodic measure $\bar{\mu}$ of $\varphi_{t}$, there exists a non-atomic ergodic measure $\mu$ of $f$ such that

$$
E_{\bar{\mu}}(\xi)=\frac{1}{E_{\mu}(\gamma)} E_{\mu}\left(\int_{0}^{\gamma(x)} \xi\left(\varphi_{t}(x, 0)\right) d t\right), \quad \forall \xi \in C^{0}(\Omega),
$$

where $E_{\bar{\mu}}(\xi)=\int_{\Omega} \xi d \bar{\mu}$ and $E_{\mu}(\gamma)=\int_{M} \gamma(y) d \mu(y)$.

The proof is similar to Lemma 4 in [7] and is thus omitted.

Corollary 2.12. If $E_{\mu}(\gamma)=+\infty$ for all non-atomic ergodic measures $\mu$ of $f$, then $\varphi_{t}$ has only atomic invariant Borel probability measures.

Proof. Otherwise, $\varphi_{t}$ should have a non-atomic invariant Borel probability measure $\bar{\mu}$ and by Lemma 2.11, there exists a non-atomic ergodic measure $\mu$ of $f$ satisfying equation (2.1). Since $\bar{\mu}$ is non-atomic, one can choose an open set $V \subset \Omega \backslash\{p\}$ such that $\bar{V} \subset \Omega \backslash\{p\}$ and $\bar{\mu}(V)>0$. Let $U$ be an open set satisfying $\bar{V} \subset U \subset \bar{U} \subset$ $\Omega \backslash\{p\}$.

Define a continuous function $\xi: \Omega \rightarrow[0,1]$ such that $\left.\xi\right|_{V} \equiv 1$ and $\left.\xi\right|_{\Omega \backslash U} \equiv 0$. Clearly, $E_{\bar{\mu}}(\xi)>0$. A straightforward calculation also shows that

$$
E_{\mu}\left(\int_{0}^{\gamma(x)} \xi\left(\varphi_{t}(x, 0)\right) d t\right)
$$

is finite. Thus $\frac{1}{E_{\mu}(\gamma)} E_{\mu}\left(\int_{0}^{\gamma(x)} \xi\left(\varphi_{t}(x, 0)\right) d t\right)=0$. This implies that $\mu$ and $\bar{\mu}$ do not satisfy equation (2.1) and so we reach a contradiction.

Lemma 2.4 will be used to show that $E_{\mu}(\gamma)=+\infty$ for $\varphi$ and then Corollary 2.12 will imply that $\varphi$ has only atomic invariant probability measures and thus has zero entropy.

2.4. Measure-theoretic entropies of $f$ and its standard suspension $\psi_{t}$. First we present a link between the invariant measures of $f$ and $\psi_{t}$.

Lemma 2.13. For any invariant probability measure $\mu$ of $f$ on $M$, we define

$$
\int_{\Omega} \xi d \bar{\mu}:=\int_{M} \int_{0}^{1} \xi(x, t) d t d \mu, \quad \forall \xi \in C^{0}(\Omega) .
$$

Then $\bar{\mu}$ is an invariant measure of $\psi_{t}$ on $\Omega$. Further, $\bar{\mu}$ is ergodic if $\mu$ is ergodic. 
The proof is elementary and omitted.

That topological entropies of a homeomorphism and its standard suspension coincide is a well-known fact; see e.g. [1. The same is true for measure-theoretic entropy, although, to our best knowledge, we could not find the original proof in the literature. For convenience of the reader we present a proof (see Proposition 2.15 below) using Katok's definition [4] of metric entropy.

For given $x \in M, n \in \mathbb{N}$ and $\varepsilon>0$, we set

$$
D(x, n, \varepsilon, f)=\left\{y \in M: d\left(f^{i} x, f^{i} y\right)<\varepsilon, i=0,1, \ldots, n-1\right\}
$$

and call it an $(n, \varepsilon, f)$-ball.

Definition 2.14 (4). Given an ergodic measure $\mu$ on $f: M \rightarrow M$ and a real $\delta \in(0,1)$, let $R(\delta, n, \varepsilon, f)$ denote the smallest number of $(n, \varepsilon, f)$-balls needed to cover a set whose $\mu$-measure is greater than $1-\delta$. Then the measure-theoretic entropy of $f$, denoted by $h_{\mu}(f)$, is defined by

$$
h_{\mu}(f):=\lim _{\varepsilon \rightarrow 0} \limsup _{n \rightarrow \infty} \frac{1}{n} \ln R(\delta, n, \varepsilon, f) .
$$

The quantity $h_{\mu}(f)$ in Definition 2.14 is independent of the choice of $\delta$ (see [4]). In a similar way we define measure-theoretic entropy for $\psi$ as follows. For $q \in \Omega$, $t \in \mathbb{R}$ and $\varepsilon>0$, we define a $(t, \varepsilon, \psi)$-ball

$$
D(q, t, \varepsilon, \psi)=\left\{w \in \Omega: d\left(\psi_{s} w, \psi_{s} q\right)<\varepsilon, 0 \leq s \leq t\right\} .
$$

Given an ergodic measure $\mu$ of $\psi$ and $\delta \in(0,1)$, let $R(\delta, t, \varepsilon, \psi)$ denote the smallest number of $(t, \varepsilon, \psi)$-balls needed to cover a set whose $\mu$-measure is greater than $1-\delta$. Then the measure-theoretic entropy of $\psi$, denoted by $h_{\mu}(\psi)$, is defined by

$$
h_{\mu}(\psi):=\lim _{\varepsilon \rightarrow 0} \limsup _{t \rightarrow \infty} \frac{1}{t} \ln R(\delta, t, \varepsilon, \psi) .
$$

One shows easily that $h_{\mu}(\psi)$ defined in this way coincides with $h_{\mu}\left(\psi_{1}\right)$, the measuretheoretic entropy for the time-one map.

Proposition 2.15. Let $\mu$ be an ergodic measure of $f$ on $M$. Then we have

$$
h_{\bar{\mu}}(\psi)=h_{\mu}(f),
$$

where $\bar{\mu}$ is defined as in Lemma 2.13 .

Proof. For given $q=(x, t) \in \Omega, n \in \mathbb{N}$ and $\varepsilon>0$, we set

$$
D(q, n, \varepsilon, \psi)=\left\{w \in \Omega: d\left(\psi_{i} w, \psi_{i} q\right)<\varepsilon, i=0,1, \ldots, n-1\right\}
$$

and

$$
\widetilde{D}(q, n, \varepsilon, \psi)=\{(y, s): y \in D(x, n, \varepsilon, f), t-\varepsilon<s<t+\varepsilon\},
$$

where $D(x, n, \varepsilon, f)$ is defined before Definition 2.14, Then by Definition 2.1 it follows that

$$
\widetilde{D}(q, n, \varepsilon, \psi)=\left\{w \in \Omega: d\left(\psi_{\tau} w, \psi_{\tau} q\right)<\varepsilon, 0 \leq \tau \leq n\right\} .
$$

We call $\widetilde{D}(q, n, \varepsilon, \psi)$ an $(n, \varepsilon, \psi)$-box with center $q$. Let $\widetilde{R}(\delta, n, \varepsilon, \psi)$ denote the smallest number of $(n, \varepsilon, \psi)$-boxes needed to cover a set whose $\bar{\mu}$-measure is greater than $1-\delta$. Then,

$$
h_{\bar{\mu}}(\psi)=\lim _{\varepsilon \rightarrow 0} \limsup _{n \rightarrow \infty} \frac{1}{n} \ln \widetilde{R}(\delta, n, \varepsilon, \psi) .
$$

We will prove that $h_{\bar{\mu}}(\psi)=h_{\mu}(f)$ in two steps. 
Step 1. For a subset $A \subset \Omega$, we denote

$$
\pi A=\{x \in M:(x, t) \in A \text {, for some } 0 \leq t<1\} .
$$

We have

$$
\bar{\mu}(A)=\int_{M} \int_{0}^{1} \chi_{A}(x, t) d t d \mu \leq \int_{M} \chi_{\pi A}(x) d \mu=\mu(\pi(A)) .
$$

Let $\varepsilon>0$ and $0<\delta<1$. If the boxes $\widetilde{D}\left(q_{1}, n, \varepsilon, \psi\right), \widetilde{D}\left(q_{2}, n, \varepsilon, \psi\right), \ldots$, $\widetilde{D}\left(q_{\widetilde{R}(\delta, n, \varepsilon, \psi)}, n, \varepsilon, \psi\right)$ cover a set $A \subset \Omega$ and $\bar{\mu}(A) \geq 1-\delta$, then $D\left(x_{1}, n, \varepsilon, f\right)$, $D\left(x_{2}, n, \varepsilon, f\right), \ldots, D\left(x_{\widetilde{R}(\delta, n, \varepsilon, \psi)}, n, \varepsilon, f\right)$ cover $\pi A$ and $\mu(\pi A) \geq 1-\delta$; here $q_{i}=$ $\left(x_{i}, t_{i}\right)$ for $i=1,2, \ldots, \widetilde{R}(\delta, n, \varepsilon, \psi)$. So, $\widetilde{R}(\delta, n, \varepsilon \psi) \geq R(\delta, n, \varepsilon, f)$ and thus $h_{\bar{\mu}}(\psi) \geq$ $h_{\mu}(f)$ by Definition 2.14 and (2.3).

Step 2. Let $\varepsilon>0$ and $0<\delta<1$. If the $(n, \varepsilon, f)$-balls

$$
D\left(x_{1}, n, \varepsilon, f\right), D\left(x_{2}, n, \varepsilon, f\right), \ldots, D\left(x_{R(\delta, n, \varepsilon, f)}, n, \varepsilon, f\right)
$$

cover $B \subset M$ with $\mu(B) \geq 1-\delta$, then the boxes

$$
\begin{array}{lll}
\widetilde{D}\left(\left(x_{1}, 0\right), n, \varepsilon, \psi\right), & \ldots, & \widetilde{D}\left(\left(x_{1}, k(\varepsilon)\right), n, \varepsilon, \psi\right), \\
\widetilde{D}\left(\left(x_{2}, 0\right), n, \varepsilon, \psi\right), & \ldots, & \widetilde{D}\left(\left(x_{2}, k(\varepsilon)\right), n, \varepsilon, \psi\right), \\
\ldots, & & \\
\widetilde{D}\left(\left(x_{R(\delta, n, \varepsilon, f)}, 0\right), n, \varepsilon, \psi\right), & \ldots, & \widetilde{D}\left(\left(x_{R(\delta, n, \varepsilon, f)}, k(\varepsilon)\right), n, \varepsilon, \psi\right)
\end{array}
$$

cover a set $A \subset \Omega$ with $\bar{\mu}(A) \geq 1-\delta$, where $k(\varepsilon)$ is the smallest integer larger than $\frac{1}{\varepsilon}$. So,

$$
\widetilde{R}(\delta, n, \varepsilon, \psi) \leq k(\varepsilon) R(\delta, n, \varepsilon, f) .
$$

Then by Definition 2.14 and (2.3) we conclude that $h_{\bar{\mu}}(\psi) \leq h_{\mu}(f)$.

\section{Proof of MAIN THEOREM}

Recall that $M$ denotes a smooth compact Riemannian manifold of dimension at least 2. In the rest of the paper we suppose that $f: M \rightarrow M$ is a $C^{\infty}$ minimal diffeomorphism, it preserves a measure $\mu$ and has positive measure-theoretic entropy $h_{\mu}(f)>0$ (and thus has a positive topological entropy). See [3] for the existence of such a diffeomorphism that preserves Riemann volume. By the variational principle we can assume that $\mu$ is an ergodic measure.

As in Definition 2.1 let $\Omega$ be the suspension manifold with the standard differentiable suspension flow $\psi: \Omega \times \mathbb{R} \rightarrow \Omega$. Denote by $X$ the $C^{\infty}$ vector field on $\Omega$ which induces $\psi$. We will construct a function $\alpha \in C^{\infty}(\Omega,[0,1])$ and a $C^{\infty}$ vector field $Y=\alpha X$ on $\Omega$ with zero topological entropy in Theorem 3.2. In Theorem 3.3 we construct a function $\widehat{\alpha} \in C^{\infty}(\Omega,[0,1])$ and corresponding $C^{\infty}$ vector field $\widehat{Y}=\widehat{\alpha} X$ on $\Omega$ with positive topological entropy. Both $\alpha$ and $\widehat{\alpha}$ will be constructed satisfying condition $(\mathbf{H})$ and by Proposition 2.2 the two differentiable flows induced by $Y$ and $\widehat{Y}$ are equivalent, thus completing the Main Theorem.

Before we prove Theorem 3.2 below, we will need the following.

Lemma 3.1. For a given sequence of positive numbers $1=\beta_{-1}>\beta_{0}>\beta_{1}>\cdots>$ $\beta_{i}>\cdots$, there exists a $C^{\infty}$ function $\omega: B^{n}(2) \rightarrow[0,1]$ such that

(1) $\omega(x)=0$ if and only if $x=0$;

(2) $\left\|\left.\omega\right|_{B^{n}\left(\frac{1}{i+1}\right)}\right\|_{C^{0}} \leq \beta_{i-1}, i=0,1,2, \ldots$;

(3) $\left.\omega\right|_{B^{n}(2) \backslash B^{n}(1)}=1$. 
Proof. Without loss of generality, we assume that $\lim _{i \rightarrow \infty} \beta_{i}=0$.

Let $h(t)$ be the function:

$$
h(t)= \begin{cases}e^{-1 / t}, & 0<t \leq 1 \\ 0, & -1<t \leq 0\end{cases}
$$

Clearly $h(t)$ is $C^{\infty}$ smooth and has zero derivatives of all orders at 0 . Let $\left\{\beta_{i}\right\}$ be as above and suppose $\left\{\alpha_{i}\right\}$ is any decreasing sequence of positive numbers $1=$ $\alpha_{-1}>\alpha_{0}>\alpha_{1}>\cdots>\alpha_{i}>\cdots$, with $\lim _{i \rightarrow \infty} \alpha_{i}=0$. For $t<\alpha_{0}$, let $\eta(t)$ be the function on $\left[-1, \alpha_{0}\right]$ defined by the series:

$$
\eta(t)=\sum_{i=1}^{\infty} 2^{-i-1} \beta_{i-1} h\left(t-\alpha_{i}\right) .
$$

This series is monotone increasing in $i$ and converges uniformly. It is zero on $[-1,0]$ and positive on $\left(0, \alpha_{0}\right]$. For any $k$ and $0<t<\alpha_{k}$ we have

$$
\eta(t)=\sum_{i=k+1}^{\infty} 2^{-i-1} \beta_{i-1} h\left(t-\alpha_{i}\right)<\frac{\beta_{k} h(1)}{2^{k}} .
$$

Further, since the derivatives of the partial sums of this series converge uniformly, we may take the derivative of the sum and we have that:

$$
\eta^{\prime}(t)=\sum_{i=1}^{\infty} 2^{-i-1} \beta_{i-1} h^{\prime}\left(t-\alpha_{i}\right) .
$$

This series is also monotone increasing and converges uniformly. For $0<t<\alpha_{k}$ we obtain

$$
\eta^{\prime}(t)=\sum_{i=j+1}^{\infty} 2^{-i-1} \beta_{i-1} h^{\prime}\left(t-\alpha_{i}\right)<\frac{\beta_{k} h^{\prime}(1)}{2^{k}} .
$$

Thus, $\lim _{t \rightarrow 0^{+}} \eta^{\prime}(t)=0$. By induction, we may also conclude that

$$
\eta^{(k)}(t)=\sum_{i=1}^{\infty} 2^{-i-1} \beta_{i-1} h^{(k)}\left(t-\alpha_{i}\right)
$$

converges uniformly and

$$
\lim _{t \rightarrow 0^{+}} \eta^{(k)}(t)=0
$$

We can now clearly extend $\eta(t)$ to the interval $[0,2]$ in such a way that $\eta$ is $C^{\infty}$ and $\eta(t)=1$ for $t \in[1,2]$.

To finish the proof of the lemma, we set $\alpha_{i}=(i+1)^{-1}$ in the above construction of $\eta(t)$ and let $\omega(x)=\eta(|x|)$ on $B^{n}(2)$. Because of the construction and equation (3.3) this function is $C^{\infty}$ smooth at 0 and on $B^{n}(2)$.

Theorem 3.2. There exists a function $\alpha \in C^{\infty}(\Omega,[0,1])$, satisfying $(\mathbf{H})$, such that the flow defined by the vector field $Y=\alpha X$ has zero topological entropy.

Proof. Recall that $p=\left[\left(x_{0}, 0\right)\right]=\pi\left(x_{0}, 0\right)$ in condition $\mathbf{( H )}$ where $\pi$ is the quotient map $\pi: M \times \mathbb{R} \rightarrow \Omega$ for the suspension.

Without loss of generality, we can assume the existence of a coordinate chart $(\widetilde{V}, \xi)$ of $\Omega$ satisfying the following.

(i) There exists an open set $V$ of $\Omega$, such that $p \in V$ and $\bar{V} \subset \widetilde{V}$.

(ii) $\xi(p)=0, \xi(V)=B^{m+1}(1), \xi(\widetilde{V})=B^{m+1}(2)$, where $2 \leq m=\operatorname{dim} M$. 
(iii) $\exists i_{1} \in \mathbb{N}$ such that

$$
\text { Closure }\left(\pi\left(B_{M}\left(x_{0}, i_{1}^{-1}\right) \times\{0\}\right)\right) \subset V
$$

and

$$
\xi\left(\pi\left(B_{M}\left(x_{0}, i_{1}^{-1}\right) \times\{0\}\right)\right) \subset \mathcal{R}=\left\{x=\left(x_{1}, \ldots, x_{m}, x_{m+1}\right): x_{m+1}=0\right\} .
$$

(iv) $\exists i_{2} \in \mathbb{N}$ such that

$$
B_{\Omega}\left(p, i_{2}^{-1}\right) \subset V
$$

and

$$
\xi\left(B_{\Omega}\left(p, i^{-1}\right)\right)=B^{m+1}\left(i^{-1}\right)
$$

for any $i_{2}<i \in \mathbb{N}$.

Under these assumptions, there exists $i_{3} \in \mathbb{N}$ and $i_{2}<i_{3}$, with the property that for any $i \geq 0$ there exists $1 \gg l_{i_{3}+i}>0$ such that

$$
\text { Closure }\left(\pi\left(B_{M}\left(x_{0}, \frac{1}{i_{3}+i}\right) \times\left[-l_{i_{3}+i}, 0\right]\right)\right) \subset B_{\Omega}\left(p, \frac{1}{i_{2}+i}\right) .
$$

We set $i_{0}:=\max \left\{i_{1}, i_{2}, i_{3}\right\}$. For any $i>i_{0}$, by Lemma 2.4, there exists $L\left(\frac{1}{i}\right)$ such that for any ergodic measure $\tau$ of $f$, we have

$$
\tau\left(B_{M}\left(f^{-1} x_{0}, \frac{1}{i}\right)\right) \geq \frac{1}{L\left(\frac{1}{i}\right)}:=\delta(i)>0 .
$$

We define $\beta_{-1}:=1$ and $\beta_{i-1}:=\frac{l_{i_{0}+i}}{i_{0}+i} \delta\left(i_{0}+i\right)$ for $i \geq 1$.

Using Lemma 3.1. one can find a $C^{\infty}$ function $\omega: \xi(\widetilde{V}) \rightarrow[0,1]$ with the properties:

(i) $\left.\omega\right|_{\xi(\tilde{V} \backslash V)} \equiv 1$

(ii) $\left\|\left.\omega\right|_{B^{m+1}\left(\frac{1}{i_{2}+i}\right)}\right\|_{C^{0}} \leq \beta_{i-1}$;

(iii) $\omega(0)=0$ and $0<\omega(a) \leq 1$ for $0 \neq a \in \xi(\widetilde{V})$.

We then define a function $\alpha$ in $C^{\infty}(\Omega,[0,1])$ as follows:

$$
\alpha(q):= \begin{cases}\omega \circ \xi(q), & q \in \widetilde{V} ; \\ 1, & q \in \Omega \backslash \widetilde{V} .\end{cases}
$$

Then

$$
\left\|\left.\alpha\right|_{B_{\Omega}\left(p, \frac{1}{i_{2}+i}\right)}\right\|_{C^{0}}=\sup _{x \in B_{\Omega}\left(p, \frac{1}{i_{2}+i}\right)}\{\alpha(x)\} \leq \beta_{i-1},
$$

where we assume, without loss of generality, that $\|\xi\|_{C^{0}} \leq 1$. We then define $Y:=$ $\alpha X$ and let $\varphi_{t}$ denote the flow induced by $Y$.

Recall the function $\gamma: M \rightarrow \mathbb{R} \cup\{\infty\}$ in Lemma 2.11 and observe that for any $x \in B_{M}\left(f^{-1}\left(x_{0}\right), \frac{1}{i_{2}+i}\right)$,

$$
l_{i_{0}+i}=\int_{t(x)}^{\gamma(x)} \sqrt{\left\langle\alpha\left(\varphi_{s}(x)\right) X\left(\varphi_{s}(x)\right), \alpha\left(\varphi_{s}(x)\right) X\left(\varphi_{s}(x)\right)\right\rangle} d s
$$


where $t(x)>0$ satisfies $\varphi_{t(x)}(x)=\psi_{1-l_{i_{0}+i}}(x)$. Then

$$
\gamma(x) \geq \gamma(x)-t(x) \geq \frac{l_{i_{0}+i}}{\left\|\left.\alpha\right|_{B_{\Omega}\left(p, \frac{1}{i_{2}+i}\right)}\right\|\|X\|} \geq \frac{l_{i_{0}+i}}{\beta_{i-1}\|X\|}=\frac{i_{0}+i}{\delta\left(i_{0}+i\right)\|X\|}
$$

for any $x \in B_{M}\left(f^{-1}\left(x_{0}\right), \frac{1}{i_{0}+i}\right)$. Therefore,

$$
\left.\gamma\right|_{B_{M}\left(f^{-1}\left(x_{0}\right), \frac{1}{i_{0}+i}\right)} \geq \frac{i_{0}+i}{\delta\left(i_{0}+i\right)\|X\|} .
$$

Now for any ergodic measure $\tau$ of $f$,

$$
E_{\tau}(\gamma)=\int_{M} \gamma(x) d \mu(x) \geq \frac{i_{0}+i}{\delta\left(i_{0}+i\right)\|X\|} \tau\left(B_{M}\left(f^{-1}\left(x_{0}\right), \frac{1}{i_{0}+i}\right)\right) \geq \frac{i_{0}+i}{\|X\|} \rightarrow+\infty
$$

as $i \rightarrow+\infty$. By Corollary 2.12, all ergodic measures of the flow $\varphi$ induced by $Y=\alpha X$ are atomic. The topological entropy $h(\varphi)$ equals $h\left(\varphi_{1}\right)$ by definition, while $h\left(\varphi_{1}\right)$ coincides with the supremum of the measure-theoretic entropies $h_{m}\left(\varphi_{1}\right)$ over all $\varphi$-invariant and ergodic measures $m$ by Theorem A of 9 (note that a $\varphi$ invariant and ergodic measure is $\varphi_{1}$-invariant but not necessarily $\varphi_{1}$-ergodic). So the topological entropy of $\varphi$ is zero.

Theorem 3.3. There exists a function $\widehat{\alpha} \in C^{\infty}(\Omega,[0,1])$, satisfying $(\mathbf{H})$, such that $\widehat{Y}=\widehat{\alpha} X$ has positive topological entropy.

Proof. Take $p \in \Omega$ as in Theorem 3.2. Recall that $f: M \rightarrow M$ is a minimal diffeomorphism which preserves an ergodic measure $\mu$ with $h_{\mu}(f)>0$. Let $\bar{\mu}$ denote the ergodic measure on $(\Omega, \psi)$ defined in Lemma 2.13. By Theorem 2.15, $h_{\bar{\mu}}(\psi)=$ $h_{\mu}(f)>0$. Noting that $\operatorname{dim} M \geq 2$ and thus that $\operatorname{dim} \Omega \geq 3$, we can find a function $\widehat{\alpha} \in C^{\infty}(\Omega,[0,1])$ that satisfies not only the assumption $(\mathbf{H})$ stated in Section 2 but also the following:

$$
\int_{\Omega} \frac{1}{\widehat{\alpha}(x)} d \bar{\mu}(x)=K<\infty .
$$

In fact, we can assume that the coordinate $(V, \xi)$ satisfies $\xi(V)=B^{m+1}(2)$ and $\xi\left(V^{\prime}\right)=B^{m+1}(1)$; here $V^{\prime}$ is an open set of $\Omega$ satisfying $p \in V^{\prime}$ and $\overline{V^{\prime}} \subset V$. We select a smooth function $\omega: \mathbb{R}^{m+1} \rightarrow \mathbb{R}$ satisfying

(1) $\omega(x)=\|x\|^{2}:=\left(x_{1}\right)^{2}+\left(x_{2}\right)^{2}+\cdots+\left(x_{m+1}\right)^{2}$, if $\|x\| \leq \frac{1}{2}$;

(2) $\frac{1}{4}<|\omega(x)|<2$, if $\frac{1}{2}<\|x\|<1$;

(3) $\omega(x)=1$, if $\|x\| \geq 1$.

It is easy to see that $\frac{1}{\omega(x)}$ is integrable w.r.t. Lebesgue measure on $B^{m+1}(2)$ since $m+1 \geq 3$. Then the function

$$
\widehat{\alpha}(q)=\left\{\begin{array}{cl}
\omega \circ \xi^{-1} & p \in V \\
1 & p \in M \backslash V
\end{array}\right.
$$

satisfies assumption (H). If $M$ is equivalent in Riemann volume, as in the example of Herman [3], then $\widehat{\alpha}$ satisfies (3.4). If $M$ has singularities, (3.4) still holds provided we choose $x_{0}$ where $M$ has finite density. By Lemma 2.6, the function

$$
\theta(t, x)=\int_{0}^{t} \frac{1}{\widehat{\alpha}\left(\psi_{s} x\right)} d s
$$


is an integrable additive function. Consider the regular set $\widehat{\Omega}$ of $\theta$ and construct the corresponding Borel $\sigma$-algebra $\widehat{B}$ (see the description after Remark 2.7). Denote by $\widehat{\varphi}_{t}$ the time changed system of $\psi_{t}$.

One can easily verify that $\widehat{\varphi}_{t}$ is the flow induced by the vector field $\widehat{Y}:=\widehat{\alpha} X$. As discussed in Section 2, we can define a measure on $(\widehat{\Omega}, \widehat{\mathcal{B}})$ :

$$
\widehat{\mu}(B)=\int_{B} d \widehat{\mu}(x)=\int_{B} \frac{1}{\widehat{\alpha}(x)} d \bar{\mu}(x)
$$

for all $B \in \widehat{\mathcal{B}}$. By Lemma 2.8, $\widehat{\mu}$ is an invariant ergodic measure of $\widehat{\varphi}_{t}$ and

$$
\widehat{\mu}(\widehat{\Omega}) \leq \int_{\Omega} \frac{1}{\widehat{\alpha}(x)} d \bar{\mu}(x)=K<\infty .
$$

Since $\bar{\mu}$ is ergodic and by Theorem 2.9 , we have

$$
h_{\widehat{\mu}}\left(\widehat{\varphi_{1}}\right) \widehat{\mu}(\widehat{\Omega})=h_{\bar{\mu}}\left(\psi_{1}\right) \bar{\mu}(\Omega)
$$

So, $h_{\widehat{\mu}}(\widehat{\varphi})=h_{\widehat{\mu}}\left(\widehat{\varphi}_{1}\right)>0$.

Proof of Theorem 1.1. The flow induced by the $C^{\infty}$ vector field $Y=\alpha X$ has zero entropy and the flow induced by the $C^{\infty}$ vector field $\widehat{Y}=\widehat{\alpha} X$ has positive entropy. They are equivalent by Proposition 2.2 .

Final note. The map $f: M \rightarrow M$ provided by [3] is real analytic. However, our method of proof clearly cannot be made analytic since $\alpha$ is flat at $p$. It seems likely that zero is an invariant of equivalent analytic flows.

\section{ACKNOWLEDGEMENT}

The authors are grateful to the referee for improving the manuscript and to David Epstein for suggesting the idea of Lemma 3.1. The second author thanks the Mathematics Research Centre at Warwick University for their hospitality during the period the work was completed.

\section{REFERENCES}

1. R. Bowen and P. Walters, Expansive one-parameter flows, J. Diff. Eq., 12 (1972), 180-193. MR 0341451 (49:6202)

2. H. Furstenburg, Poincaré recurrence and number theory, Bull. A.M.S. 5 (1981), 211-234. MR628658 (83d:10067)

3. M.R. Herman, Construction d'un diffeomorphisme minimal d'entropie topologique non nulle, Erg. Th. Dyn. Systems, 1 (1981), 65-76. MR627787 (83c:58046)

4. A. Katok, Lyapunov exponents, entropy and periodic orbits for diffeomorphisms, Publ. IHES, 51 (1980), 137-173. MR.573822 (81i:28022)

5. S.T. Liao, On the stability conjecture, Chinese Ann. Math. 1 (1980), no. 1, 9-30. MR591229 (82c:58031)

6. G. Maruyama, Theory of stationary processes and ergodic theory, A lecture at the Symposium held at Kyoto Univ., 1965.

7. T. Ohno, A weak equivalence and topological entropy, Publ. RIMS, Kyoto Univ. 16 (1980), 289-298. MR574037 (81g:54053)

8. W. Sun and E. Vargas, Entropy of flows, revisited, Bol. Soc. Brasil. Mat. 30 (1999), 315-333. MR 1726916 (2001d:37007)

9. W. Sun, Entropy of orthonormal $n$-frame flows, Nonlinearity, 14 (2001), no. 4, 829-842. MR.1837641 (2002e:37032)

10. R. Thomas, Topological entropy of fixed-point free flows, Trans. Amer. Math. Soc., 319 (1990), 601-618. MR1010414 (90m:58178) 
11. R. Thomas, Entropy of expansive flows, Erg. Th. Dyn. Systems, 7 (1987), 611-625. MR.922368 (89a:54022)

12. H. Totoki, Time changes of flows, Mem. Fac. Sci. Kyushu Univ., Ser. A, 20 (1966), 27-55. MR 0201606 (34:1488)

13. P. Walters, An introduction to ergodic theory, Springer-Verlag, 1982. MR648108 (84e:28017)

Lmam, School of Mathematical Sciences, Peking University, Beijing, People's RePUBLIC OF CHINA

E-mail address: sunwx@math.pku.edu.cn

Department of Mathematics, Ohio University, Athens, Ohio 45701

E-mail address: young@math.ohiou.edu

School of Mathematical Sciences, Peking University, Beijing, People's Republic of China

E-mail address: zhouyh@math.pku.edu.cn 